American Journal of Infectious Diseases 2 (3): 153-158, 2006

ISSN 1553-6203

(c) 2006 Science Publications

\title{
The Effects of Growth Hormone Therapy in HIV-infection
}

\author{
Donald P. Kotler \\ Gastrointestinal Division, Department of Medicine, St. Luke's-Roosevelt Hospital Center \\ College of Physicians and Surgeons, Columbia University, New York
}

\begin{abstract}
The results of these studies suggested that the growth hormone might play a role in the management of visceral fat accumulation associated with HIV. Analysis of the treatment effect sizes in the published studies show that the effects of various doses of rhGH on trunk fat fit a dose-response characteristic.
\end{abstract}

Key words: HIV, malnutrition, HIV lipodystrophy, visceral fat, body composition, growth hormone

\section{INTRODUCTION}

Nutritional alterations are common in HIV infection. The types of nutritional abnormalities encountered in clinical practice differ as a function of antiretroviral therapy. Nutritional alterations in the preHAART era were characterized by disproportionate losses of lean body mass and skeletal muscle. Nutritional alterations in patients on HAART more commonly demonstrate fat redistribution and metabolic alterations without depletion of lean mass, a condition termed lipodystrophy ${ }^{[1]}$. Growth hormone therapy has been applied in both situations, though with different rationales. At the time of publication of this review, growth hormone is FDA approved for the treatment of AIDS wasting and is not FDA approved for the treatment of HIV-associated lipodystrophy or related conditions.

Effect of growth hormone therapy in HIV-associated wasting: Recombinant human growth hormone (rhGH) has been applied in HIV-infected individuals for the last 12 years. The original application was as a treatment for HIV-associated wasting, which was defined as an involuntary weight loss of greater that $10 \%$ of body weight. The rationale for therapy was that HIVassociated wasting was associated with substantial depletion of body cell mass ${ }^{[2]}$, while treatment with hypercaloric feedings, whether by oral, enteral, or parenteral routes, was associated with a predominant gain in fat and no restoration of body cell mass ${ }^{[3]}$. A 12 week, randomized, double-blind, placebo-controlled trial of rhGH, using a pharmacologic dose of $6 \mathrm{mg}$ per day, demonstrated significant weight gain, that was comprised of about a 3 kilogram gain in lean mass associated with a fall of just under $2 \mathrm{~kg}$ of fat, the latter measurements made by a variety of body composition techniques ${ }^{[4]}$. Thus, measurement of weight change underestimated the anabolic effect that accompanies rhGH therapy. About one half of the gain was in extracellular volume while one half was in intracellular volume, the latter representing the true anabolic effect. The increase in lean mass was associated with the increase in work output, as measured by maximum voluntary effort on a treadmill. A subsequent study did not confirm a significant increase in body weight compared to placebo therapy ${ }^{[5]}$. Unfortunately, body composition studies were not performed in that study. However, therapy was found to be associated with improvements in quality of life. Gelato and colleagues studied AIDS patients with wasting syndrome and noticed that there was resistance to the anabolic effects of rhGH, thus providing a rationale for the supraphysiologic doses being used ${ }^{[6]}$.

The effectiveness of rhGH as therapy of HIVassociated wasting was reevaluated in the HAART era by performing a randomized, double-blind, placebocontrolled trial ${ }^{[7]}$. Once again, wasting was defined as a weight loss $\geq 10 \%$. The results of this study, which compared $6 \mathrm{mg}$ daily to $6 \mathrm{mg}$ on alternate days, also showed an increase in lean body mass and a decrease in body fat by DXA scanning and by multi-frequency bioelectrical impedance analysis (Fig. 1), as well as an improvement in mean exercise output, in this study done by cycle ergometery. This study also documented improvements for quality of life in response to rhGH therapy. While the patients in this study had much higher CD4 lymphocyte counts than those in the previous study ${ }^{[4]}$, subjects in both studies had undergone comparable weight loss, in most cases accompanied by low levels of lean body mass. The dose of $6 \mathrm{mg}$ daily led to greater increases in lean body mass than the same dose given on alternate days, though both doses had similar effects on exercise capacity.

The use of rhGH in the treatment of HIVassociated wasting has diminished as the impact of this complication has declined sharply in the face of HAART therapy. Cost considerations in relation to expected benefits also have limited its use clinically. Its application also has been confounded by a lack of a strict definition of wasting. There is evidence that the criterion of an involuntary weight loss of $\geq 10 \%$ is

Corresponding Author: $\quad$ Donald P. Kotler, MD, GI Division / S \& R 1301, St. Luke's-Roosevelt Hospital Center, 1111 Amsterdam Avenue, New York, NY 10025, Tel: 212523-3670, Fax: 212523-3678 


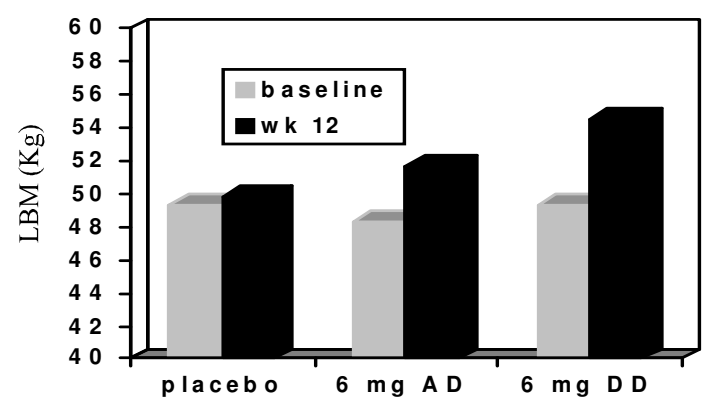

Fig. 1:Effect of rhGH on lean body mass, as determined by multifrequency bioelectrical impedance analysis, in HIV-infected subjects with $\geq 10 \%$ weight $\operatorname{loss}^{[7]}$. Both $6 \mathrm{mg} /$ day and $6 \mathrm{mg}$ on alternate days led to an increase in lean body mass compared to placebo, with a greater effect seen at the higher dosage. $\mathrm{N}=203$ placebo, $\mathrm{N}=206$ rhGH on alternate days, $\mathrm{N}=178 \mathrm{on} 6 \mathrm{mg}$ rhGH daily, $\mathrm{p}<0.001$

neither sensitive nor specific for detecting protein energy malnutrition ${ }^{[8,9]}$

Effect of rhGH given during acute opportunistic infections: Studies in both the pre-HAART and HAART eras have evaluated the use of rhGH during acute opportunistic infections, with the rationale that such therapy might decrease the acute loses of lean mass that occur during disease complications. Paton and colleagues studied $20 \mathrm{HIV}$ - infected patients with pneumocystis pneumonia, cytomeglovirus infection, or serious bacterial infections ${ }^{[10]}$. Subjects received $6 \mathrm{mg}$ per day of rhGH for 2 weeks, then were followed for an additional 4 weeks. Therapy significantly increased lean body mass and decreased body fat, while the placebo had no effect. Some positive effects on grip strength and quality of life were noted. However, the benefits disappeared soon after therapy was discontinued.

Schambelan and colleagues also evaluated the use of rhGH during acute infections, in this case in the HAART era and in comparison to the effects of a cytokine inhibitor, thalidomide ${ }^{[11]}$. Subjects received 6 $\mathrm{mg}$ per day for 4 weeks and then were followed for a total of 8 weeks. The results of this study showed that rhGH-treated subjects had an insignificant increase in body weight compared to baseline results, but significant increases in lean body mass and decreases in body fat contents. In contrast, thalidomide had no significant effects on body composition. No significant differences in viral load, CD4 lymphocyte count, or serious adverse events were found in the $\mathrm{rhGH}$, thalidomide, or placebo groups.

Growth hormone and visceral fat content: HIVassociated lipodystrophy is a constellation of signs and symptoms, which include an alteration of body fat distribution, notably a decrease in the content of subcutaneous fat and an increase in the sizes of the visceral and other upper body adipose tissue compartments, plus the frequent coexistence of dyslipidemia and insulin resistance ${ }^{[12]}$. Visceral fat accumulation is seen in about $30 \%$ of patients. While the triad of visceral fat accumulation, dyslipidemia and insulin resistance are well appreciated in general medicine, where they are often known as the metabolic syndrome, their sudden appearance during the therapy of a viral infection was unexpected. Their presence has led to great concern about the possibility of accelerated atherosclerosis in affected patients and investigations are underway to specifically examine this possibility ${ }^{[13]}$.

The results of many studies indicate that growth hormone secretion and the content of visceral adipose tissue act in a mutually antagonistic manner. Growth hormone deficiency whether by trauma, tumor, surgery or congenital deficiencies all are associated with accumulation of visceral fat content and replacement therapy reduces visceral fat content ${ }^{[14,15]}$. In addition growth hormone secretion, as measured by overnight sampling methods, is low in non-HIV infected patients $^{[16]}$. In such subjects, replacement therapy also reduces visceral fat content. There are multiple associations between growth hormone deficiency and cardiovascular risk beyond simple visceral fat accumulation. Patients with growth hormone deficiency also have been show to be insulin resistant, to have dyslipidemia, an increased prevalence of hypertension, elevated levels of prothrombotic factors in plasma and indeed, evidence of accelerated atherosclerosis ${ }^{[17]}$.

Johannsen and colleagues evaluated the effect of growth hormone therapy in men with abdominal obesity. They performed a prospective, randomized, double-blind, placebo-controlled trial in 30 men with a waist-to-hip ratio of $>0.95^{[16]}$. The subjects were treated with either growth hormone at $1 \mathrm{mg}$ per day, or placebo, for 9 months. Measurements were made at baseline, 6 weeks, 6 months and 9 months. The investigators found that GH treated subjects remained weight stable, though body fat decreased by $9 \%$ and lean mass rose. The losses of visceral fat were greater, in relative terms, than the losses of subcutaneous fat. Insulin resistance was increased by 6 weeks after the initiation of therapy, but returned to baseline values by 9 months. Therapy also was associated with a decrease in diastolic blood pressure. Of note, adverse events were noted in about half of patients and appeared related to fluid retention.

Studies of growth hormone in HIV-associated lipodystrophy: Reitschel and colleagues evaluated overnight growth hormone in HIV-infected men with and without lipodystrophy, as well as in controls ${ }^{[18]}$. Growth hormone secretion was lower in the HIVinfected group than in the controls. Furthermore, growth hormone secretion varied inversely with 
visceral fat content. The difference in growth hormone secretion was shown to be related to a decrease in pulse amplitude, rather than pulse frequency. These results suggest that the interrelationships between visceral fat and growth hormone secretion are similar in HIVinfected and non-HIV infected individuals.

A series of investigator-initiated studies were performed and the results reported between 1999- 2002. Torres and colleagues noted an effect of growth hormone on patients with buffalo hump using a dose of $6 \mathrm{mg}$ per day ${ }^{[19]}$. Wanke and colleagues ${ }^{[20]}$, Nguyen and colleagues $^{[21]}$ and Mauss and colleagues ${ }^{[22]}$ also demonstrated decreases in trunk fat and visceral fat in response to $\mathrm{rhGH}$ doses of $6 \mathrm{mg}$ per day. Lo and colleagues $^{[23]}$ evaluated the effects of $3 \mathrm{mg}$ per day, both on body composition and insulin resistance and corroborated Johannsen's finding ${ }^{[16]}$ that insulin resistance rose early in the course of treatment, then returned to baseline with prolonged therapy.

Engelson and colleagues studied the effects of rhGH at both $6 \mathrm{mg}$ per day and $4 \mathrm{mg}$ every other day ( 2 $\mathrm{mg}$ per day) on body composition, insulin resistance and serum lipids during 6-month treatment periods ${ }^{[24]}$. The dose of $6 \mathrm{mg}$ per day led to a $45 \%$ drop in visceral fat content along with a $10 \%$ gain in lean mass. Side effects were frequent and mostly related to fluid retention. However, $10 \%$ of the study subjects developed diabetes and a number of other adverse events were noted, including the diagnosis of cancers in 3 subjects, though any relationship of the cancers to rhGH use is speculative. In a sub-study of this trial, He and his colleagues examined the relationship between the insulin resistance, as measured by the integrated insulin concentrations during oral glucose tolerance tests and subcompartments of visceral fat ${ }^{[25]}$. The investigators noted that omental fat drains into the portal vein and the liver, whereas retroperitoneal fat drains into the inferior vena cava and hypothesized that there also may be metabolic difference between the 2 subcompartments. The baseline contents of omental/mesenteric fat and retroperitoneal fat were determined separately and compared to measures of insulin resistance by multiple regression. Omental/mesenteric fat but not retroperitoneal fat was statistically related to insulin resistance.

Based on these preliminary findings, a randomized, phase II/III, multi-center, double-blind, placebocontrolled trial of rhGH was undertaken ${ }^{[26]}$. The target population for this study was the subgroup of HIVlipodystrophy characterized by visceral fat accumulation, termed HIV Adipose Redistribution Syndrome, or HARS. The study, which initially involved about 240 subjects, included three randomizations. The first randomization was a 12-week comparison of $4 \mathrm{mg} / \mathrm{day}, 4 \mathrm{mg}$ every other day and placebo. The primary endpoints of this study were visceral fat content, as determined by single slice CT scanning at the level of L4-L5 and trunk/limb fat ratio, as determined by dual $\mathrm{X}$ ray absorptiometry (DXA). Secondary endpoints included other body composition measures, parameters of glucose and lipid metabolism and quality of life. A second randomization, covering from 12-24 weeks, provided pilot information about possible long term strategies and included drug discontinuation from $4 \mathrm{mg} /$ day, dose reduction from 4 $\mathrm{mg}$ to $2 \mathrm{mg} / \mathrm{day}$ and drug continuation at $2 \mathrm{mg} / \mathrm{day}$, while subjects initially receiving placebo were given 4 mg/day. After another 12 weeks in which all subjects received growth hormone, a third randomization was performed and subjects received either $1 \mathrm{mg}$ or $2 \mathrm{mg}$ per day of rhGH for an additional 6 months as an expanded trial of maintenance therapy.

Eligibility criteria included documented HIV infection in the presence of HAART. For screening purposes, excess VAT was determined by anthropometric criteria; in men a waist circumference greater than $88.3 \mathrm{~cm}$ and a waist-to-hip ratio greater than 0.95 , in women, a waist circumference greater than $75.3 \mathrm{~cm}$ and a waist-to-hip ratio greater than 0.90 . The subjects could not be diabetic, or even glucose intolerant on oral glucose tolerance testing. In contrast, therapy with lipid-lowering agents was permitted. The average age of the group was about 45 and about $13 \%$ of subjects were female. The mean CD4+ lymphocyte count was in the mid 400s, while the mean body mass index was about 27, the latter representing about $20 \%$ body fat. There was marked increased in trunk fat and in visceral fat the latter about 4 times than seen in a control population ${ }^{[27]}$.

The results of this study showed that both doses of rhGH led to a statistically significant decrease in visceral fat on CT scanning, (Fig. 2a). Significant decreases in trunk fat and trunk-to-limb fat ratio also were seen at both doses of rhGH. As expected, the relative and absolute losses of trunk fat were greater than the losses of limb fat.

After the second randomization, further studies showed significant regain of visceral fat with dose interruption and retention of the therapeutic effects with dose reduction and dose continuation. Observations to week 60, after the third randomization, in subjects receiving either $1 \mathrm{mg}$ or $2 \mathrm{mg}$ per day showed a significant net effect of between $1-1.5 \mathrm{~kg}$ of trunk fat, compared to baseline values.

Safety analyses demonstrated significant improvements in both total and non-HDL cholesterol concentrations in patients receiving $4 \mathrm{mg}$ daily and 4 $\mathrm{mg}$ on alternate days. The benefits were maintained with dose reductions and dose continuation, but not with drug discontinuation and significant decreases in total and non-HDL cholesterol were maintained in subjects receiving either $1 \mathrm{mg}$ or $2 \mathrm{mg}$ per day. Studies of glucose metabolism showed that both $4 \mathrm{mg}$ daily and $4 \mathrm{mg}$ of rhGH on alternate days significantly increased both fasting and 120 minute glucose and insulin concentrations. As seen with other studies, the increase 


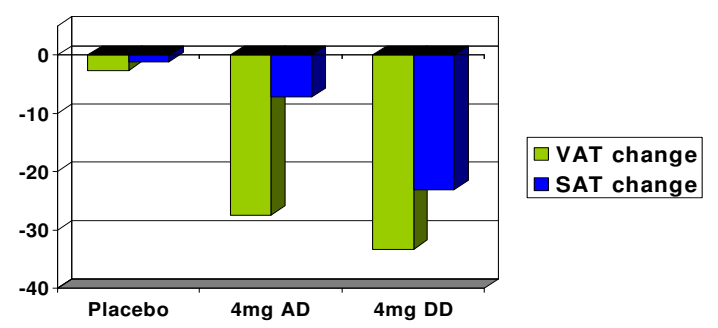

Fig. 2a: Effect of rhGH on body fat distribution, as determined by single slice CT scans, in HIVinfected individuals with $\mathrm{HARS}^{[26]}$. Doses of 4 $\mathrm{mg}$ daily and on alternate days led to significant losses of both VAT $(\mathrm{p}<0.001)$ and SAT ( $<<0.001$ for $4 \mathrm{mg}$ DD and $\mathrm{p}<0.05$ for 4 mg AD), compared to placebo therapy. $\mathrm{N}=53$ placebo, $\mathrm{N}=56$ on $4 \mathrm{mg}$ on alternate days, $\mathrm{N}=59$ on $4 \mathrm{mg}$ daily. Data as change $\mathrm{ncm}^{2}$.

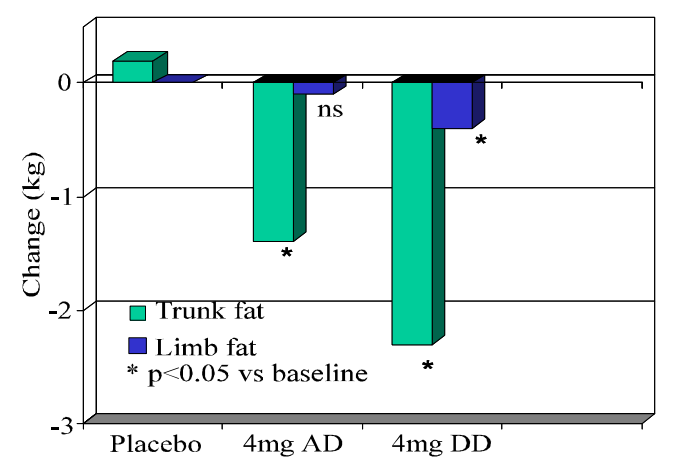

Fig. 2b: Effect of rhGH on trunk and limb fat, as determined by DXA scanning ${ }^{[26]}$. Both $4 \mathrm{mg}$ daily and on alternate days led to a decrease in trunk fat, with the daily dose also promoting loss of limb fat, as compared to baseline. The observed effects were greater with the higher dose of rhGH. $\mathrm{N}=76$ placebo, $\mathrm{N}=79$ on $4 \mathrm{mg}$ on alternate days, $\mathrm{N}=82 \mathrm{on} 4 \mathrm{mg}$ daily

in insulin resistance found by 12 weeks had disappeared by the 6-month follow-up.

This study also systematically evaluated quality of life. A measurement of distress related to body image found that the appearance of the belly was the most discouraging body site to the subjects who entered the trial. While rhGH therapy at $4 \mathrm{mg}$ per day decreased the level of distress from all parts of the body, except for the face, the measured improvement in the appearance of the belly was the greatest, quantitatively. The level of distress related to the appearance of the belly also

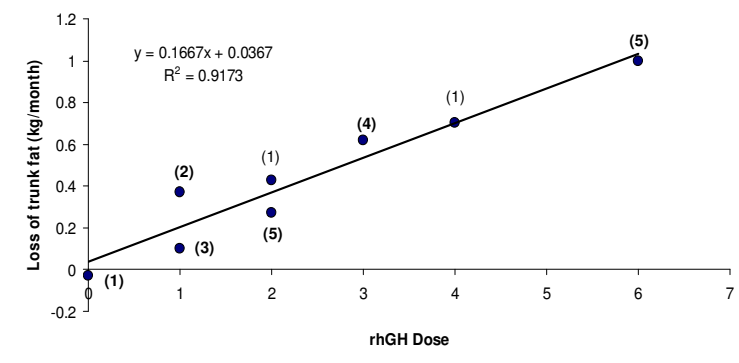

Fig. 3: Effect of different doses of rhGH on trunk fat, as determined by DXA scanning and expressed as $\mathrm{kg}$ change/month. This graph is a compilation of data from several studies. Numbers in parentheses represent the various published studies ${ }^{[1,23,24,26,28,29]}$

decreased significantly on the $4 \mathrm{mg}$ alternate day dose. A second registration trial is underway.

Recently, two studies utilizing $1 \mathrm{mg}$ doses of rhGH have been published and have shown some body composition and metabolic effects ${ }^{[28,29]}$.

Effect of growth hormone releasing factor: A phase 2, double-blind, placebo-controlled, study of a growth hormone releasing factor analog for 12 weeks has been reported ${ }^{[30]}$. The rationale is that growth hormone, while effective in reducing visceral fat content and serum cholesterol concentrations, it has toxicity including increased insulin resistance as well as elevated concentrations of serum IGF-1, with worries about long term adverse outcomes. Growth hormone releasing factor is more physiologic than growth hormone in that it allows for feedback inhibition at the level of the pituitary gland. On the other hand, feedback inhibition might limit the benefits of therapy.

The eligibility criteria for this study were similar as for the studies of rhGH. The drug was administered at two doses, $1 \mathrm{mg}$ and $2 \mathrm{mg}$. The results of this study demonstrated a significant fall in trunk fat of $1.4 \mathrm{~kg}$, by DXA scanning. In contrast, the change in visceral fat content did not reach statistical significance when compared to placebo, though it did when compared to baseline values. In contrast to $\mathrm{rhGH}$, there was no significant rise on insulin resistance with therapy. While serum IGF-1 concentrations increased significantly, mean values remained within the normal range. There were no changes in quality of life, though abdominal symptoms decreased. 


\section{CONCLUSION}

In addition to questions and safety and efficacy, there are three major unresolved issues. The first has to do with definitions that are clinically applicable methods of diagnosing visceral fat accumulation as well as monitoring its response during treatment. However, it is clear we are not just treating a body compartment but rather a factor related to cardiovascular risk but an understanding of how to put this information in a management scheme is elusive a present. In the event that therapy is chosen, the optimal induction dose still needs definition, as does the overall question of whether or not induction is needed in the face of longterm maintenance therapy. Finally, the appropriate maintenance dose for long-term therapy needs to be defined.

\section{REFERENCES}

1. Carr, A., K. Samaras and S. Burton et al., 1998. A Syndrome of peripheral lipodystrophy, hyperlipidaemia and insulin resistance in patients receiving HIV protease inhibitors. AIDS, 12: F51-8.

2. Kotler, D.P., J. Wang and R.N. Pierson, 1998. Studies of body composition in patients with the acquired immunodeficiency syndrome. Am. J. Clin. Nutr., 42: 1255-65.

3. Kotler, D.P., 2000. Nutritional alterations associated with HIV infection. J. Acquir. Immune. Defic. Syndr., 25: S81-7.

4. Schambelan, M., K. Mulligan, C. Grunfeld, E.S. Daar, A. Lamarca, D.P. Kotler, J. Wang, S.A. Bozzette and J.B. Breitmeyer, 1996. Recombinant human growth hormone in patients with HIVassociated wasting. Ann. Intern. Med., 125: 873882.

5. Berger, D.S., A. Lamarca, H. Landy, R.S. Kauffman and J. Breitmeyer, 1996. A phase III study of recombinant human growth hormone (mammalian cell-derived) in patients with AIDS wasting. XI Intl. Conf. AIDS, 1: 26.

6. Frost, R.A., C.H. Lang and M.C. Gelato, 1997. Growth hormone/insulin-like growth factor axis in human immunodeficiency virus-associated disease. Endocrinologist, 7: 23-31.

7. Moyle, G.J., E. Daar, J.M. Gertner, D. Kotler, J.C. Melchior, F. O'Brien and E. Svanberg, 2004. Growth hormone improves nutritional status, physical performance and quality of life in subjects with HIV-associated wasting on HAART. J. Acquir. Immun. Defic. Syndr., 35: 367-75.

8. Wheeler, D.A., C.L. Gibert and C.A. Launier et al., 1998. Weight loss as a predictor of survival and isease progression in HIV infection. J. Acquir. Immun. Defic. Syndr., 18: 80-5.
9. Maia, B., E.S. Engelson, J. Wang and D.P. Kotler. 2005. Antiretroviral therapy affects the composition of weight loss in HIV infection: implications for clinical nutrtition. Clinical Nutrition 24: 971-8.

10. Paton, N.I., P.J. Newton, D.R. Sharpstone, H.M. Ross, J. Cotton, A.G. Calder, E. Milne, M. Elisa, S. Shah, P. Engrand, D.C. Macallan, B.G. Gazzard and G.E. Griffin, 1999. Short term growth hormone administration at the time of opportunistic infections in HIV-positive patients. AIDS, 13: 1195-1202.

11. Schambelan, M., K. Mulligan, P. Bacchetti, K. Johansen, S. Bhasin, C. Grunfeld, D.P. Kotler and S. Hulley, 2003. Safety and efficacy of short-term treatment with growth hormone and thalidomide in HIV-infected patients with a secondary infection: A randonized, double-blind, placebo-controlled trial. Proc. 10th Conf. on Retroviruses and Opportunistic Infections, Abstract 791.

12. Kotler, D.P., 2003. HIV infection and lipodystrophy. Prog. Cardiovasc. Dis., 45: 269-84.

13. Friis-Moller, N., C.A. Sabin and R. Weber et al., 2003. Combination antiretroviral therapy and the risk of myocardial infarction. N. Engl. J. Med., 349: 1993-2003.

14. Lönn, L., G. Johannsson and L. Sjöström et al., 1996. Body composition and tissue distribution in growth hormone deficient adults before and after growth hormone treatment. Obes. Res., 4: 45-54.

15. Snel, Y.E.M., R.-J.M. Brummer and M.E. Doerga et a1., 1995. Adipose tissue assessed by magnetic resonance imaging in growth hormone-deficient adults: the effect of growth hormone replacement and a comparison with control subject. Am. J. Clin. Nutr., 61: 1290-4.

16. Johannson, G., P. Mårin and L. Lönn et al., 1997. Growth hormone treatment of abdominally obese men reduces abdominal mass, improves glucose and lipoprotein metabolism and reduces diastolic blood pressure. J. Clin. Endocrinol. Metab., 82: 727-34.

17. Colao, A., C. di Somma and A. Cuocolo et al., 2001. Improved cardiovascular risk factors and cardiac performance after 12 months of growth hormone $(\mathrm{GH})$ replacement in young adult patients with GH deficiency. J. Clin. Endocrinol. Metab., 86: 1874-81.

18. Rietschel, P., C. Hadigan and C. Corcoran et al., 2001. Assessment of Growth Hormone Dynamics in Human Immunodeficiency Virus- Related Lipodystrophy. J. Clin. Endocrinol. Metab., 86: 504-10.

19. Torres, R.A., K.W. Unger, J.A. Cadman and J.Y. Kassous, 1999. Recombinant human growth hormone improves truncal adiposity and 'buffalo humps' in HIV-positive patients on HAART [letter]. AIDS, 13: 2479-81. 
20. Wanke, C., J. Gerrior, J. Kantaros, E. Coakley and M. Albrecht, 1999. Recombinant human growth hormone improves the fat redistribution syndrome (lipodystrophy) in patients with HIV. AIDS, 13: 2099-103.

21. Nguyen, Q.V., R. Malinverni and H. Furrer, 2000. Treatment of HAART associated fat accumulation disease with recombinant human growth hormone: Results of a randomised double blind placebo controlled crossover trial. XIII Intl. AIDS Conf., Abstracts.

22. Mauss, S., E. Wolf and H. Jaeger, 1999. Reversal of protease inhibitor_related visceral abdominal fat accumulation with recombinant human growth hormone [letter]. Ann. Intern. Med., 131: 313-4.

23. Lo, J.C., K. Mulligan, M.A. Noor, J.-M. Schwarz, R.A. Halvorsen, C. Grunfeld and M. Schambelan, 2001. The effects of recombinant human growth hormone on body composition and glucose metabolism in HIV-infected patients with fat accumulation. J. Clin. Endocrinol. Metab., 86: 3480-7.

24. Engelson, E.S., M.J. Glesby, D. Mendez, J.B. Albu, J. Wang, S.B. Heymsfield and D.P. Kotler, 2002. Effect of recombinant human growth hormone in the treatment of visceral fat accumulation in HIV infection. J. Acquir. Immun. Defic. Syndr., 30: 379-91.

25. He, Q., E.S. Engelson, D.P. Kotler, J.B. Albu and S.B. Heymsfield, 2003. Preferential loss of omental-mesenteric adipose tissue during growth hormone therapy of HIV-lipodystrophy. J. App. Physiol., 94: 2051-7.
26. Kotler, D.P., N. Muurahainen, C. Grunfeld, C. Wanke, M. Thompson, M. Saag, D. Bock, J.M. Gertner and the STARS study group, 2004. Effects of growth hormone on abnormal visceral adipose tissue accumulation and dyslipidemia in HIVinfected patients. J. Acquir. Immun. Defic. Syndr., 35: 239-54

27. Saag, M., D. Lee, C. Wanke, J. Gertner, E. Engelson, S. Heymsfield and D. Kotler. Physical characteristics of patients with human immunodeficiency virus-associated adipose redistribution syndrome (HARS). Proc. 44th ICAAC. Abstract H-161.

28. Anderson, O., S.B. Haugaard and A. Flyvbjerg et al., 2004. Low-dose growth hormone and human immunodeficiency virus-associated lipodystrophy syndrome: A pilot study. Eur. J. Clin. Invest., 34: 561-8.

29. Lo, J.C., K. Mulligan and M.A. Noor et al., 2004. The effects of low-dose growth hormone in HIVinfected men with fat accumulation. A pilot study. Clin. Infect. Dis., 39: 732-5.

30. Falutz J., S. Allas, D. Kotler, B. Lussier, M. Thompson, J. Albu, L. Vacheron, T. Aribat, S. Grinspoon. 2005. A placebo-controlled, dose ranging study of a growth hormone releasing factor in HIV-infected patients with abdominal fat accumulation. AIDS; 19:1279-87. 